\title{
Creation and Structure of Baby Universes in Monopole Collisions
}

\author{
Arvind Borde $^{a * \dagger}$, Mark Trodden ${ }^{b \ddagger}$ and Tanmay Vachaspati ${ }^{b \S}$ \\ ${ }^{a}$ Institute of Cosmology \\ Department of Physics and Astronomy \\ Tufts University \\ Medford, MA 02155, USA. \\ ${ }^{b}$ Particle Astrophysics Theory Group \\ Department of Physics \\ Case Western Reserve University \\ 10900 Euclid Avenue \\ Cleveland, OH 44106-7079, USA.
}

\begin{abstract}
Under certain circumstances, the collision of magnetic monopoles, topologically locked-in regions of false vacuum, leads to topological inflation and the creation of baby universes. The future evolution of initial data represented by the two incoming monopoles may contain a timelike singularity but this need not be the case. We discuss the global structure of the spacetime associated with monopole collisions and also that of topological inflation. We suggest that topological inflation within magnetic monopoles leads to an eternally reproducing universe.
\end{abstract}

CWRU-P25-98

LIU-SC-TCSG-1/1998001

Typeset in REVTEX

*borde@cosmos2.phy.tufts.edu

${ }^{\dagger}$ Permanent address: Theoretical and Computational Studies Group, Natural Science Division, Southampton College of Long Island University, Southampton, NY 11968, USA.

\footnotetext{
$\ddagger_{\text {trodden@theory1.phys. cwru.edu }}$

$\S_{\text {txv7@po.cwru.edu }}$
} 


\section{INTRODUCTION}

The inflationary universe scenario provides a natural mechanism by which an initially small region of space can expand exponentially in a short time. The particular implementation of inflation in which we are interested is topological inflation. Exponential expansion occurs here within the cores of topological defects such as monopoles, vortices, or domain walls [1,2]. In this scenario, the formation of defects in a phase transition soon after the big bang is accompanied by inflation within the defects, provided certain parameters assume values in suitable ranges. We are interested in this paper in the dependence of topological inflation on particle physics parameters and, in particular, on the topological winding of the defect, since it is possible to change the winding by bringing together several defects. In the case of vortices [3] (see also [⿴囗十), there exists a range of parameters for which the conditions for topological inflation are satisfied for high winding vortices, but not for low winding ones. Can the conditions that are necessary for inflation to take place be satisfied today if we merge small winding defects to produce a larger winding defect? If so, we could, in principle, create a "baby universe" in the laboratory.

In general, topological inflation occurs if the width $w$ of a topological defect is larger than the horizon size corresponding to the energy density $\rho_{V}$ in the core of the defect:

$$
w>\left(\frac{3}{8 \pi G \rho_{V}}\right)^{1 / 2}
$$

which, for unit winding defects, is typically satisfied for symmetry breaking scales $\eta$ larger than the Planck mass $m_{p}$. We refer to defects that do not satisfy the inequality (1.1) as subcritical, and those that do as supercritical.

An intuitive understanding of why higher topological charges alleviate the high symmetry breaking scales required for topological inflation can be obtained by considering the asymptotic form of the metric for a static cosmic string solution aligned with the $z$-axis [5]:

$$
d s^{2}=d t^{2}-d r^{2}-d z^{2}-r^{2} d \tilde{\theta}^{2}
$$


Here $(r, \tilde{\theta}, z)$ are cylindrical polar coordinates in a locally Minkowski but globally conical spatial section, with, for critical coupling, $\tilde{\theta}$ taking values in the range

$$
0 \leq \tilde{\theta}<2 \pi\left(1-4|n| \mu_{1}\right)
$$

where $n$ is the topological winding number of the string, and $\mu_{1} \sim \eta^{2}$ is the energy per unit length of a string with unit winding (in Planck units). This static metric is applicable as long as the deficit angle is less than $2 \pi$ and hence static solutions cease to exist for

$$
\eta \gtrsim \frac{m_{p}}{2 \sqrt{|n|}}
$$

Thus, the critical scale at which asymptotically static solutions become impossible decreases with increasing winding as $1 / \sqrt{n}$. Of course, the absence of static solutions does not guarantee that the core will inflate. A numerical study [3] (see also [4]) shows, however, that topological inflation does set in at critical symmetry breaking scales with approximately this dependence on $n$.

These results imply that one could start with several non-inflating $n=1$ vortices and combine them to form a large winding vortex which would then start inflating. In this paper we argue that a similar process holds for magnetic monopoles. Further, in certain models, colliding magnetic monopoles in a regular, asymptotically flat spacetime can lead to the creation of a region of space that satisfies all of the conditions for inflation to occur - that is, it has the inflationary equation of state in a sufficiently large spatial volume. This is our "baby universe". Farhi and Guth [6] have, however, pointed out that the future development of such a spacetime is likely to be marred by singularities. Their result rests on a theorem of Penrose [7] that states that a final (initial) singularity must occur in a spacetime in which there exists a trapped (anti-trapped) surface, as long as

1. $R_{\mu \nu} k^{\mu} k^{\nu} \geq 0 \forall$ null $k^{\mu}$ (where $R_{\mu \nu}$ is the Ricci tensor), and

2. the spacetime contains a noncompact Cauchy surface. 
A trapped (anti-trapped) surface here is a closed 2-surface for which both the ingoing and outgoing sets of light rays normal to the surface are converging (diverging) at every point on the surface.

Application of the Penrose theorem to the collision of monopoles means that, under its assumptions, a singularity must be present to the past of any region containing anti-trapped surfaces. It follows that we are unlikely to be able to evolve initial data to the point where we can find anti-trapped surfaces (such as occur in de Sitter space) without first encountering a singularity. This is the "obstacle" to the creation of an actual baby universe in the laboratory discussed by Farhi and Guth.

Singularities can be avoided, however, in spacetimes in which the assumptions of Penrose's theorem are violated. In such spacetimes, the future evolution of the expanding baby universe is regular. Assumption (1) follows in Einstein's theory from the weak energy condition (which says that the matter energy density must be positive as measured by any observer). The assumption is violated if we allow negative matter energy densities, or if we look at certain alternate theories of gravitation, in which there are extra terms in the field equation that allow assumption (1) to be violated even when the weak energy condition holds. Examples of such theories are some higher derivative gravity models [8], and dilatoninspired scalar-tensor models [9]. In these models the singularity is avoided because of the existence of a limiting length, such as one might expect if string theory were the underlying physics. We do not consider the violation of assumption (1) any further in this paper.

Assumption (2) is a very strong one and there are examples of solutions to Einstein's equation (with reasonable matter) that violate it [10]. Without the assumption, several scenarios are possible [11]. The one that is of greatest use to us, since we are interested in creating regions of de Sitter space, has a closed Universe forming to the future of the trapped surface. This scenario will become clear in Sec. [1] when we draw the Penrose diagrams for the spacetime in which two non-inflating monopoles collide to form a higher winding monopole within which the conditions for topological inflation are met.

In earlier work [12 14] monopoles undergoing topological inflation have been studied by solving the classical equations of motion. The initial conditions are chosen to mimic 
cosmological conditions and the numerical results reveal that the core of the monopole expands exponentially. In Sec. III we consider the large-scale structure of spacetime for the cosmological scenario of topological inflation within magnetic monopoles and construct spacetime diagrams. Several important features of these diagrams are forced on us by general theorems on global spacetime structure. Some of the claims in [12] are inconsistent with our diagrams. We believe that there is an error in that work in the computation of the behavior of null geodesics, as we explain in Sec. [II].

In Sec. IV we discuss the specific example of monopoles in an $S U(5)$ theory, and show that there is a region of parameter space in which the unit winding monopoles do not satisfy the conditions for topological inflation but the higher winding monopoles do. The discussion in this section is meant to provide an example in which spherically symmetric monopoles of various windings can exist and be stable. Our results on the spacetime of inflating monopoles from the previous sections are valid more generally.

In the concluding section we point out the possibility that the inflating region of spacetime may detach from the asymptotic region. If this is true, it is possible that the detached baby universe may split into three other universes which can then each split into three more, and so on, ad infinitum. Then the monopole core may contain a very large number of baby universes and not just one. Finally, we also point out the possible relevance of topological inflation to models in which there is a duality between particles and solitons.

\section{SPACETIME STRUCTURE FOR BABY UNIVERSE CREATION}

Consider the spacetime evolution as two monopoles with unit winding $(n=1)$ collide and coalesce . We assume that the $n=1$ monopoles are not inflating. Hence the metric external

\footnotetext{
${ }^{1} \mathrm{~A}$ rich variety of gravitating magnetic monopole solutions are known and it has been proposed that it may be possible to understand these using catastrophe theory [15]. For a given set of parameters, there can be more than one solution to the Einstein-Yang-Mills equations, but only one solution is stable. Here we shall always assume that we are working with the stable solution for any given set of parameters.
} 
to the $n=1$ monopoles is Reissner-Nordstrom and can be continued smoothly within the interiors of the monopole cores. Thus, at early times the spacetime is asymptotically flat and there are no singularities. When the monopoles collide and coalesce, the result is an $n=2$ monopole, which we assume satisfies the conditions for topological inflation. (In Sec. IV we will show that this is possible in certain models.) However, the condition for topological inflation necessarily requires that the monopole be a black hole [1]. To see this note that the black hole condition is that the width of the monopole be less than the associated Schwarzschild radius

$$
w \lesssim 2 G m
$$

with $m$ the monopole mass. (We are assuming that $\rho_{V}$ is large and hence the magnetic charge of the monopole is small compared to its mass in natural units.) Now, we may estimate the mass of the monopole by assuming that the core has constant energy density $\rho_{V}$, which yields $m \sim 4 \pi \rho_{V} w^{3} / 3$. Inserting this into (2.1) gives

$$
w \gtrsim\left(\frac{3}{8 \pi G \rho_{V}}\right)^{1 / 2},
$$

which means that the condition for a monopole to undergo topological inflation is identical to the condition that it is a black hole as seen from the exterior region.

Are there singularities in this spacetime? Since the black hole conditions are satisfied, the spacetime is likely to have trapped surfaces and hence it would appear from Penrose's theorem that there must be a singularity to the future of the initial data (incoming $n=1$ monopoles). Also, if the interior of the monopole is to inflate, the spacetime there must become approximately de Sitter and, since de Sitter space has anti-trapped surfaces, it would again appear that there must be a singularity on which at least one past directed null geodesic originating in the interior terminates. Thus it would seem that there are two singularities in the spacetime. An important point here, however, is that the singularity that lies to the future of the asymptotic Reissner-Nordstrom region can be the same singularity as lies to the past of the de Sitter region Thus, the "crunch" of the black hole can play the role of the big bang for the inflating region. 


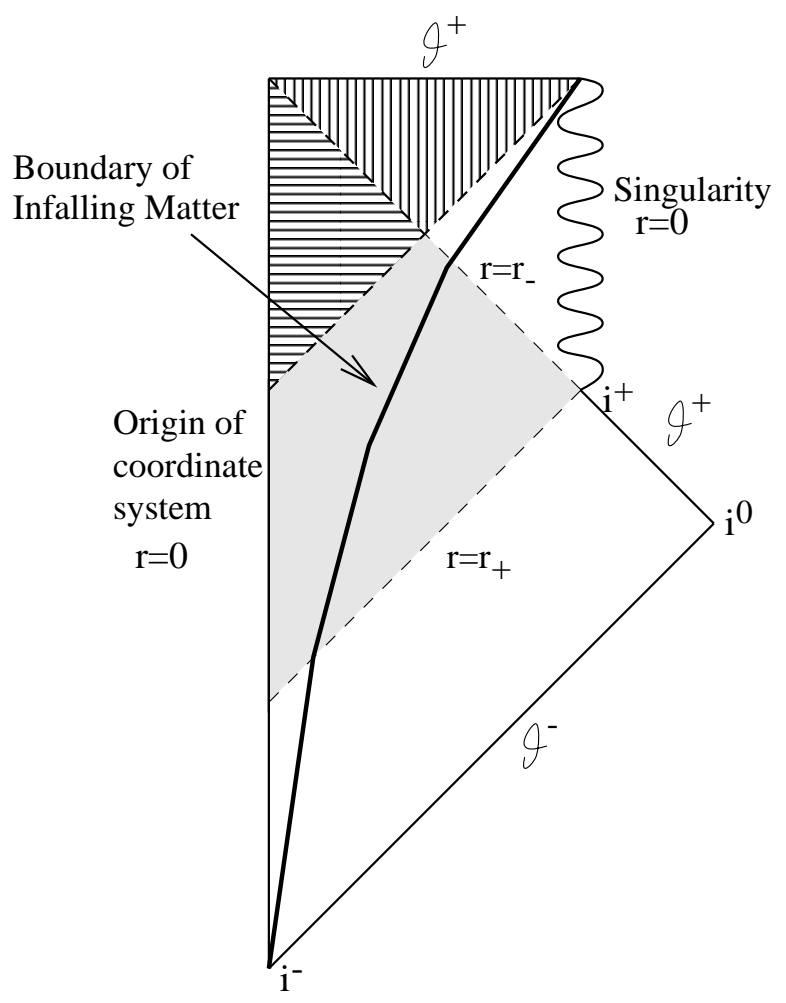

FIG. 1. Penrose diagram for a singular spacetime as two subcritical $n=1$ monopoles collide and produce a supercritical $n=2$ monopole, the interior of which then satisfies the conditions for topological inflation and is a candidate baby universe. The supercritical monopole is necessarily a black hole. The surface $r=r_{+}$is the event horizon. The infalling matter lies in the region between the origin of the coordinate system and the thick curve.

In Fig. 1 we show the spacetime structure of the inflating monopoles when there is an "initial-final" singularity of this type 2 . Each point in the interior of this diagram represents a spacelike 2-sphere. The horizon structure is exactly that of part of the Reissner-Nordstrom spacetime. In the horizontally shaded region, outgoing future directed null rays escape to future null infinity $\left(\mathcal{J}^{+}\right)$even though they are inside the monopole. The equation of state in this region is inflationary $(p=-\rho)$, and we can time evolve our initial data (the wellseparated $n=1$ monopoles) to make predictions about this region. Note, however, that there are no anti-trapped surfaces in the horizontally shaded region, since those would be inconsistent with the Penrose theorem. The anti-trapped surfaces appear in the vertically

\footnotetext{
${ }^{2}$ The spacetime is similar to that shown in Hawking and Ellis [10] (page 361) for the collapse of a charged dust cloud to form a Reissner-Nordstrom black hole.
} 
shaded region. Incoming and outgoing null rays directly expand here to null infinity. To the past of this region, as suggested by Farhi and Guth, there is a singularity. Predictability in this region is lost due to signals originating at the singularity and at $i^{+}$. However, if boundary conditions at the timelike singularity and infinity can be imposed, predictability will be restored. Trapped surfaces occur in the region shaded with light gray, and the singularity to their future is the same as the one to the past of the anti-trapped surfaces.

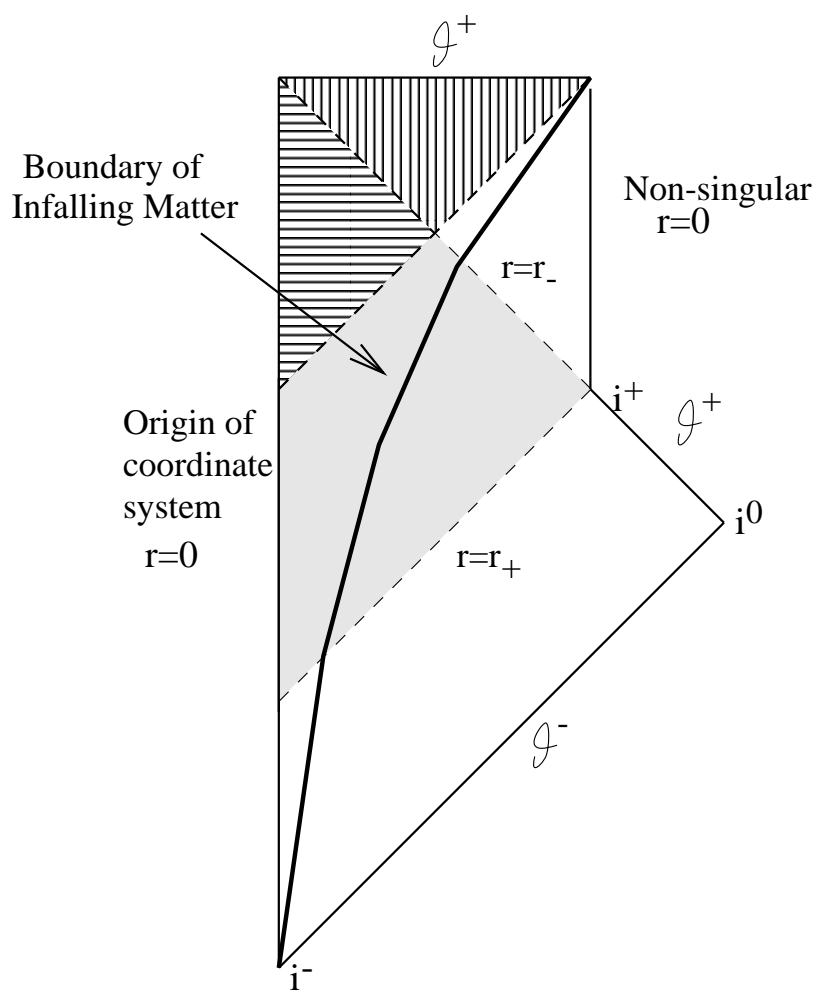

FIG. 2. Penrose diagram for a nonsingular spacetime as two subcritical $n=1$ monopoles collide and produce a supercritical $n=2$ monopole, the interior of which then satisfies the conditions for topological inflation and is a baby universe. As in Fig. 1, the supercritical monopole is necessarily a black hole and the surface $r=r_{+}$is the event horizon.

Is this singularity necessary? In Einstein's theory with "normal" matter, the singularity is required to occur if there is a noncompact Cauchy surface. The example in Fig. 1 does not possess such a surface (the vertically shaded region, for example, lies outside the Cauchy development of any asymptotically flat, initial value hypersurface). It is known that singularities can be avoided in such scenarios [11]. We then have a spacetime structure like the one in Fig. 2. The global structure here is similar to that of Fig. 1 (and the differently 
shaded regions have the same meanings), except that there are no singularities. This means that a spacelike slice between one $r=0$ origin of coordinates and the other $r=0$ line must be a 3-sphere. In other words a closed Universe evolves out of our initially open one. In this case predictability is lost due to signals originating at $i^{+}$.

It has been shown under very general assumptions [16,11] that these are the only two possibilities: we must either have singularities or we must have topology change.

\section{COSMOLOGICAL TOPOLOGICAL INFLATION}

In contrast to the monopole collision scenario that we discussed previously, the standard picture of topological inflation is that the phenomenon occurs in the extremely early universe. In this case, it is reasonable to ask if the initial singularity that may be associated with baby universe production is in fact the usual big bang singularity. If so, the presence of antitrapped surfaces would not require any additional singularities. (The presence of trapped surfaces, as seen from the asymptotic region may, of course, still lead to a future singularity.)

A careful look at the possibilities does not, however, seem to permit us to unify the usual big bang singularity with any that might be associated with baby universe production. Consider, for example, an attempt to glue a de Sitter region to the interior of a ReissnerNordstrom black hole between $r_{-}$and $r_{+}$, the inner and outer horizons. This will mean that there are anti-trapped 2-surfaces in the de Sitter region whose past does not intersect the usual Reissner-Nordstrom singularity. Then the only singularity that might be associated with our baby universe is the usual big bang one. Such a spacetime is schematically depicted in Fig. 3. There are trapped surfaces in the region shaded with light gray, and de Sitter antitrapped surfaces in the region with vertical shading. (The determination of what surfaces are trapped or anti-trapped is done via purely local calculations of the expansion of null geodesics, and thus depends solely on the local metric and not on the global properties of the spacetime.) The double vertical line shown is the singularity that might exist associated with the trapped surfaces (although, topology change would allow us to avoid this singularity, as we've mentioned earlier). There is also a limiting case of this scenario in which the de 
Sitter region is glued to the Reissner-Nordstrom spacetime along $r_{+}$. There are no trapped surfaces here and no Reissner-Nordstrom singularity.

The properties of these spacetimes are, however, self-contradictory. Consider an antitrapped surface $S_{1}$ whose past does not intersect the Reissner-Nordstrom singularity, and consider another surface $S_{2}$, a "normal" 2-sphere just outside the horizon, to the past of $S_{1}$. We expect such surfaces $S_{2}$ close to the horizon to behave as they do in the usual ReissnerNordstrom case and to be unaffected by cosmological considerations. In-going light rays from $S_{2}$ are thus converging, but when they reach the region that contains $S_{1}$ they must be diverging without yet having focused. This can only occur if the weak energy condition is violated.

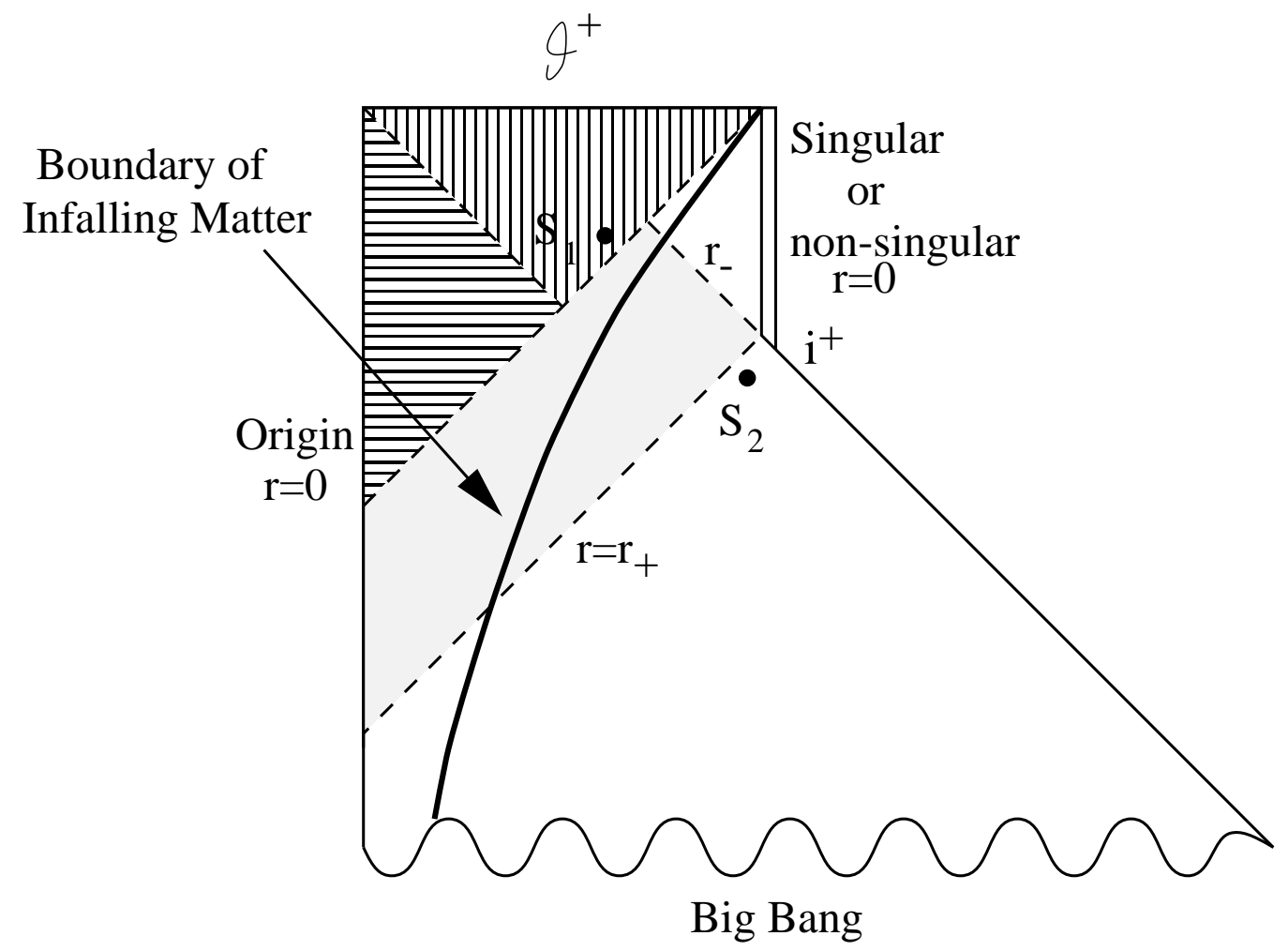

FIG. 3. A possible Penrose diagram for the spacetime as a supercritical topological defect undergoes topological inflation in the very early universe. For reasons explained in the text, this is not a viable diagram.

The only possibility appears to be the one shown in Fig. 4 . Again, there are trapped surfaces in the region shaded with light gray, and anti-trapped surfaces in the region with 
vertical shading. The singularity shown is the one that might exist associated with both the trapped and the anti-trapped surfaces (although, topology change would allow you to avoid this singularity here as well). There is also a separate cosmological singularity.

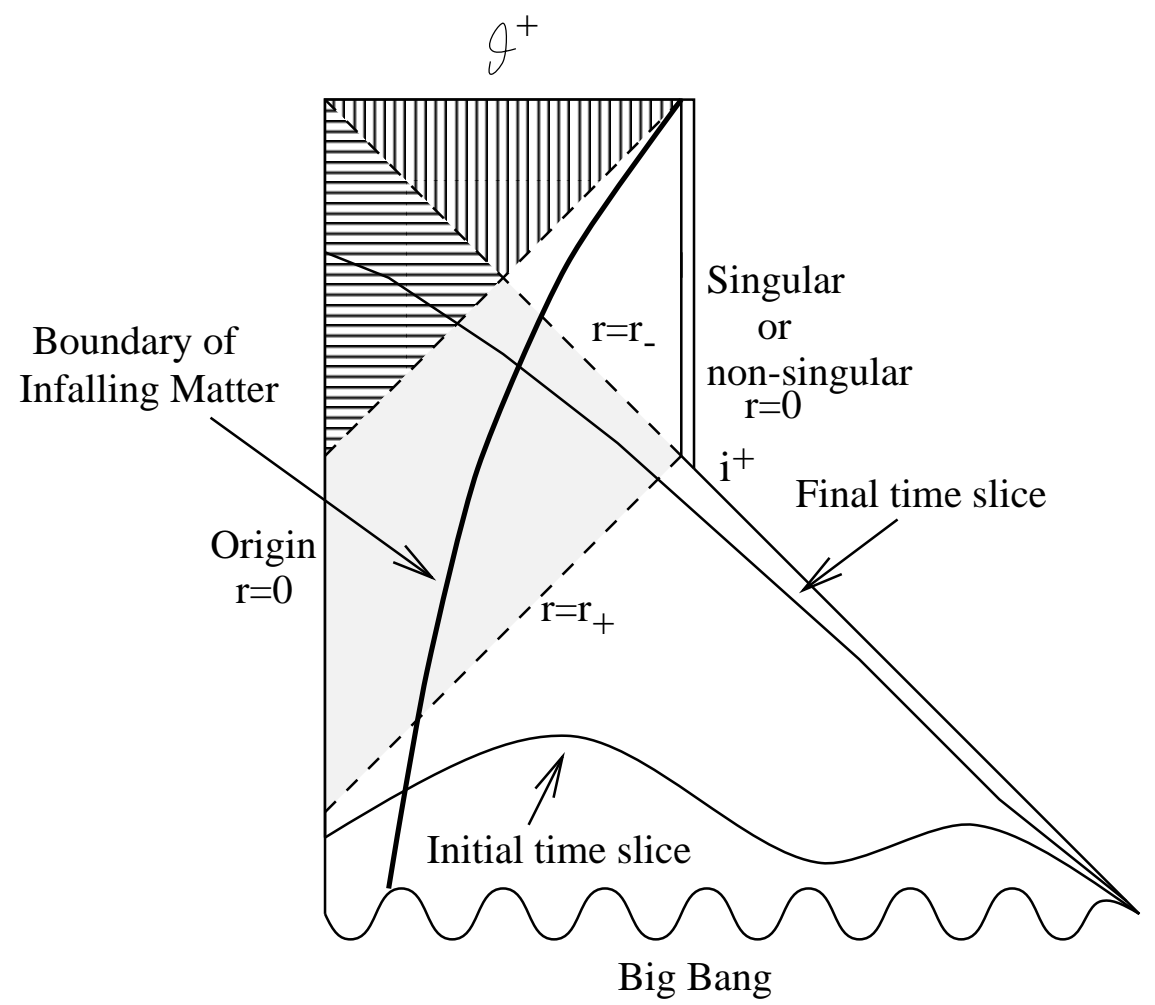

FIG. 4. Penrose diagram for the spacetime as a supercritical topological defect undergoes topological inflation in the very early universe.

In Fig. 6 we also show a spacelike hypersurface on which one can specify initial data and then evolve numerically until the Reissner-Nordstrom singularity develops or the topology change occurs. Such an evolution has been studied in [12,[13]. It has been claimed in [12] that the evolution can be followed all the way until anti-trapped surfaces form. This might be possible if the global spacetime structure were like the one shown in Fig. [3. But, as we have

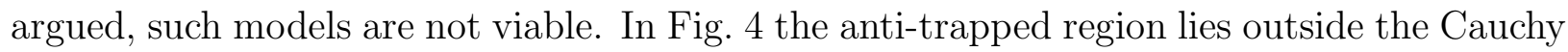
development of the initial time slice, and no final time slice can intersect it. The horizon structure claimed in [12] is also more complicated than ours. The horizon structure and the claim that anti-trapped surfaces can be seen to form are both based on a computation of the expansion of radial null geodesics at different points in spacetime. There appears to be 
an error, however, in the computation of the expansion in [12, which throws into question the entire discussion there of horizons ${ }^{3}$.

\section{A SPECIFIC MODEL: $S U(5)$ MONOPOLES AND TOPOLOGICAL INFLATION}

Consider the familiar Grand Unified symmetry breaking scheme

$$
G \equiv S U(5) \rightarrow \frac{S U(3) \times S U(2) \times U(1)}{\mathcal{Z}_{6}} \equiv H_{S M}
$$

realized by the vacuum expectation value (VEV) of an $S U(5)$ adjoint Higgs field. If we write [19] the potential of the Higgs as

$$
V(\Phi)=-m_{1}^{2}\left[\operatorname{Tr}\left(\Phi^{2}\right)\right]+a\left[\operatorname{Tr}\left(\Phi^{2}\right)\right]^{2}+b \operatorname{Tr}\left(\Phi^{4}\right)
$$

with $m_{1}$ a mass scale and $a, b$ dimensionless parameters, then the appropriate VEV is

$$
\langle\Phi\rangle=v_{1} \operatorname{diag}(2,2,2,-3,-3)
$$

with

$$
v_{1} \equiv \frac{m_{1}}{\sqrt{60 a+14 b}}
$$

It is known [17,18] that the resulting spectrum of stable monopole solutions consists of those with topological windings $n=1,2,3,4,6$. External to any monopole solution, the symmetry group of the vacuum is $H_{S M}$. However, the symmetry group in the core $H_{\text {core }}^{(n)}$ depends on the topological charge. We shall focus on the following cases:

$$
\begin{aligned}
& H_{\text {core }}^{(1)}=S U(2) \times S U(2) \times U(1) \\
& H_{\text {core }}^{(2)}=S U(4) \times U(1)
\end{aligned}
$$

\footnotetext{
3 The metric in [12] is $d s^{2}=-d t^{2}+A^{2}(t, r) d r^{2}+B^{2}(t, r) r^{2}\left(d \theta^{2}+\sin ^{2} \theta d \phi^{2}\right)$. The radial null vector field $N^{\mu}=\left(-1, \pm A^{-1}, 0,0\right)$ is used in that reference in order to determine trapped and antitrapped surfaces. But $N^{\mu}$ is not the tangent vector associated with affinely parametrized radial null geodesics, and so its expansion, $\theta \equiv N_{; \mu}^{\mu}$, cannot be used to find trapped and anti-trapped surfaces.
} 
In the broken phase, where the gauge group is that of the standard model, we may decompose $\Phi$ into three pieces, transforming as $(\mathbf{8}, \mathbf{1}),(\mathbf{1}, \mathbf{3}),(\mathbf{1}, \mathbf{1})$, representations of $\mathrm{SU}(3) \times \mathrm{SU}(2)$, with masses (following the notation of [19])

$$
\mu_{8} \equiv \sqrt{20 b} v_{1}, \quad \mu_{3} \equiv 2 \mu_{8}, \quad \mu_{0} \equiv 2 m_{1}
$$

respectively.

The potentially interesting regime for us is one in which the following conditions are satisfied:

1. The correct SU(5) symmetry breaking occurs.

2. The $n=1$ monopoles can attract to form an $n=2$ monopole.

These criteria are satisfied by the following parameter choices. The symmetry breaking occurs if we choose

$$
\mu_{3}=2 \mu_{8}
$$

and the $n=1$ monopoles attract for

$$
2 \mu_{8}>\mu_{0}
$$

Now, the condition (1.1) that a monopole inflates, may be expressed in terms of our mass parameters as

$$
\left(\frac{8 \pi G \rho_{V}}{3}\right)^{1 / 2}=H>\mu_{3}=2 \mu_{8}
$$

We are interested in the possibility that, within the parameter range we have specified, the $n=2$ monopoles might satisfy (4.9) but the $n=1$ monopoles might not. We may calculate the energy densities $\rho_{V}^{(n)}$ in the cores of monopoles of winding $n$. In the regime above, the relevant monopoles satisfy

$$
\begin{aligned}
& \rho_{V}^{(1)}=\frac{m_{1}^{4}}{4 b}\left[\left(\frac{1}{x+7 / 30}\right)-\left(\frac{1}{x+\alpha_{1}}\right)\right] \\
& \rho_{V}^{(2)}=\frac{m_{1}^{4}}{4 b}\left[\left(\frac{1}{x+7 / 30}\right)-\left(\frac{1}{x+\alpha_{2}}\right)\right],
\end{aligned}
$$


where

$$
\alpha_{1}=\left(\frac{8}{35}\right)^{2}\left(\frac{113}{16}+\frac{2}{256}\right), \quad \alpha_{2}=\frac{16}{25}
$$

and $x \equiv a / b$. From these expressions it is easy to see that $\rho_{V}^{(1)}<\rho_{V}^{(2)}$ for a suitable choice of parameters. Thus, $S U(5)$ monopoles provide a natural particle physics setting in which our scenario could operate.

\section{CONCLUSIONS}

We have investigated a novel mechanism for producing a baby universe in the laboratory. In particular, we have shown that, in a certain range of parameters, magnetic monopoles can collide to produce a spacetime structure which is a Reissner-Nordstrom black hole from the original asymptotic region, but has an interior with the inflationary equation of state. This baby universe will begin to expand, but the extent to which we may predict its later evolution is unclear. In one scenario, there exists a singularity and the future evolution of the baby universe is unpredictable. In another scenario there is no spacetime singularity, and a closed baby universe develops. The future evolution of the baby universe is unpredictable here as well.

One possible development of the spacetime [20] is that the inflating region pinches off the asymptotic spacetime, leading to a disconnected baby universe. It is useful to picture this

process using an embedding diagram. To construct the embedding diagram, we suppress one spatial dimension and consider the resulting $2+1$ dimensional spacetime at a fixed time $t$. We then construct the induced metric on a rotationally invariant spatial 2-surface $z=f(r)$, embedded in 3+1 dimensional Minkowski space. This metric is:

$$
d s^{2}=\left(1+f^{\prime 2}\right) d r^{2}+r^{2} d \theta^{2}
$$

where, $f^{\prime}$ denotes the derivative of $f$ with respect to $r$. For a certain choice of function $f$, and for a suitably defined radial coordinate $R(r)$, this metric will be identical to the truncated metric of our spacetime. The surface $z=f(r)$ then gives the embedding diagram 


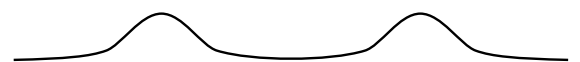

(a)

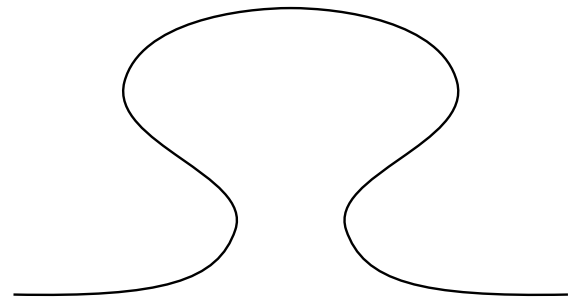

(c)

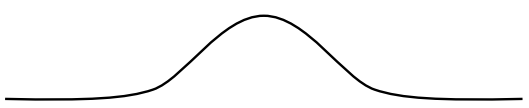

(b)
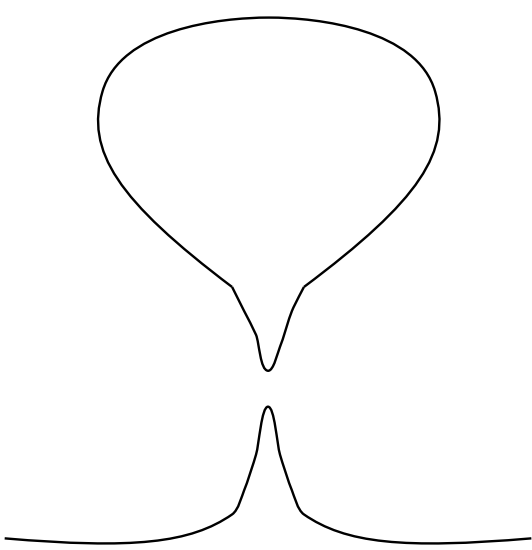

(d)

FIG. 5. Embedding diagrams showing the incoming unit winding monopole, their coalescence to form a higher winding, inflating monopole, and the development of a pinch that disconnects the baby universe from the mother universe.

at different times. The behaviour of the embedded surface with time is shown schematically in Fig. 5. (Parts (b) and (c) of Fig. 5 correspond to the embedding diagrams of Sakai [12].)

The possibility that the baby universe disconnects from the original spacetime leads to further questions. What happens to the magnetic charge in the original spacetime? Since the asymptotic magnetic field is left intact by the pinching off, we conclude that the singularity must be accompanied by the pair creation of a magnetic monopole and antimonopole. The magnetic monopole stays on at the Reissner-Nordstrom singularity of the asymptotic spacetime while the antimonopole is attached to the singularity at the "south pole" of the detached baby universe. This is also consistent with Gauss' law since the total magnetic charge of the closed (baby) universe must vanish. But now the baby universe with a monopole at the north pole and the antimonopole at the south pole, inflates. Eventually, the equatorial region will be inflated far away from the polar regions. In this situation, the northern (and southern) hemispheres are exactly like the spacetime shown in Fig. 5b. Further development must lead to inflation of the polar cap regions, ultimately leading to their pinching off, yielding two new universes in addition to the original baby universe 
(see Fig. 6). Since this process must continue forever, it leads to an eternally reproducing universe.

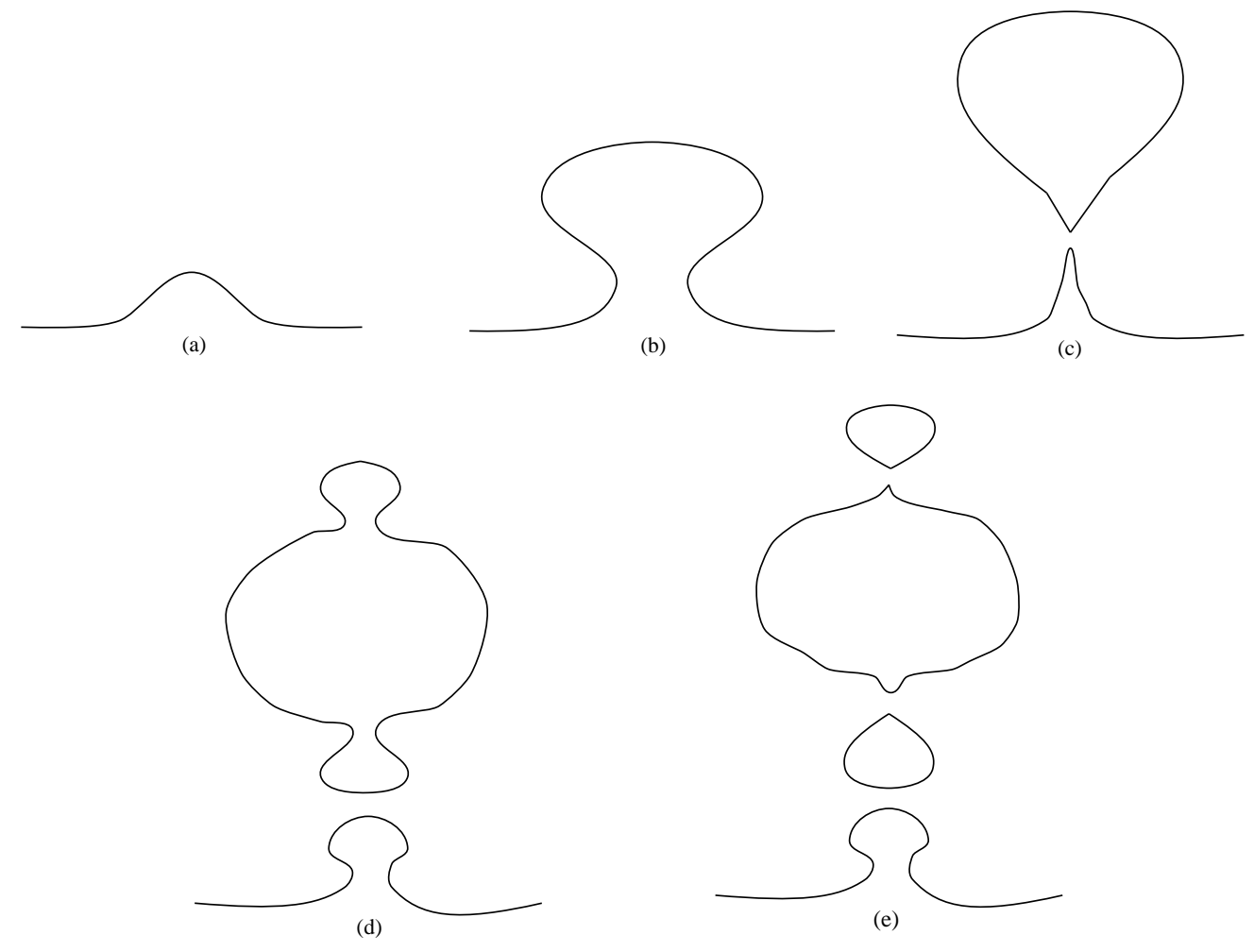

FIG. 6. Embedding diagrams showing the subdivision of the baby universe.

To conclude, the collisions of heavy monopoles may lead to the scenario described in this paper. However, the creation of a baby universe cannot be observed by its creator since signals from within the baby universe cannot reach the asymptotic region.

Another possibility arises if the particles that we observe in nature are in fact the magnetic monopoles of another dual theory [21, 19]. In that case, it is possible that stellar collapse into black holes can lead to the production of a baby universe, since such a collapse corresponds to the coalescence of about $10^{57}$ particles. An important issue in this scenario is that the net electric charge of a star is zero and so it corresponds to the coalescence of monopoles and antimonopoles with zero net winding. However, if all that is required for inflation is the occurrence of a large region of false vacuum, even the collision of monopoles and antimonopoles might provide the right conditions. 


\section{ACKNOWLEDGMENTS}

We thank Glenn Starkman, Cyrus Taylor and Alex Vilenkin for useful discussions. This work was supported by the Department of Energy (D.O.E.). M.T. is also supported by

the National Science Foundation (N.S.F.). A.B. thanks the Research Awards Committee of Southampton College for its financial support and the Institute of Cosmology at Tufts University and the High Energy Theory Group at Brookhaven National Laboratory for their hospitality. 


\section{REFERENCES}

[1] A. Vilenkin, Phys. Rev. Lett. 72, 3137 (1994).

[2] A. Linde, Phys. Lett. B327, 208 (1994).

[3] A. de Laix, M. Trodden and T. Vachaspati, Phys. Rev. D57, 7186 (1998).

[4] I. Cho, "Inflation and Nonsingular Spacetimes of Cosmic Strings", gr-qc/9804086.

[5] A. Vilenkin, Phys. Rev D23, 852 (1981); J.R. Gott, Ap. J. 288, 422 (1985); W.A. Hiscock, Phys. Rev. D31, 3288 (1985).

[6] E. Farhi and A. Guth, Phys. Lett. B183, 149 (1987).

[7] R. Penrose, Phys. Rev. Lett. 14, 57 (1965).

[8] V. Mukhanov and R. Brandenberger, Phys. Rev. Lett. 68, 1969 (1992); R. Brandenberger, V. Mukhanov and A. Sornborger, Phys. Rev. D48, 1629 (1993).

[9] M. Trodden, V. Mukhanov, R. Brandenberger, Phys. Lett. B316 483 (1993); R. Moessner and M. Trodden, Phys. Rev. D51, 2801 (1995).

[10] S.W. Hawking and G.F.R. Ellis, "The large-scale structure of space-time", Cambridge University Press (1973).

[11] A. Borde, Phys. Rev. D55, 7615 (1997).

[12] N. Sakai, Phys. Rev. D54, 1548 (1996)

[13] N. Sakai, H. Shinkai, T. Tachizawa and K. Maeda, Phys. Rev. D53, 655 (1996); Erratum-ibid D54, 2981 (1996).

[14] I. Cho, A. Vilenkin, Phys. Rev. D56, 7621 (1997).

[15] T. Tachizawa, K. Maeda and T. Torii, Phys. Rev. D51, 4054 (1995).

[16] A. Borde, Phys. Rev. D50, 3692, (1994).

[17] C. Gardner and J. Harvey, Phys. Rev. Lett. 52, 879 (1984).

[18] C. Gardner, Phys. Lett. B142, 379 (1984).

[19] H. Liu and T. Vachaspati, Phys. Rev. D56, 1300 (1997).

[20] S. Blau, E. Guendelman and A. Guth, Phys. Rev. D35, 1747 (1987).

[21] T. Vachaspati, Phys. Rev. Lett. 76, 188 (1996). 